Review

\title{
A Review on Ultra Reliable and Low Latency Communications (PHY and MAC Layer Perspectives)
}

\author{
Prinu Chacko Philip and Mohammed Abdel-Hafez \\ Department of Electrical Engineering, United Arab Emirates University, Al Ain, Abu Dhabi, UAE
}

\author{
Article history \\ Received: 17-06-2020 \\ Revised: 20-07-2020 \\ Accepted: 05-08-2020 \\ Corresponding Author: \\ Prinu Chacko Philip \\ Department of Electrical \\ Engineering, United Arab \\ Emirates University, Al Ain, \\ Abu Dhabi, UAE \\ Email: 201990050@uaeu.ac.ae
}

\begin{abstract}
Fifth Generation (5G) wireless network plays major attention in the communication scenario due to the advanced features in data transmission. $5 \mathrm{G}$ is designed to overcome the issues, such as data rate, reliability, throughput, latency and capacity in the wireless network domains. The $5 \mathrm{G}$ wireless services aim to increase the data rate through massive communication services. In this survey, well known research papers based on low latency communication at the Physical layer (PHY) and Medium Access Control layer (MAC) are reviewed based on the UltraReliable Low Latency Communication (URLLC) perspective. Here, the communication techniques are categorized as, structure-based model, resource reuse-based techniques, radio resource management techniques, scheduling based approach, diversity-based techniques and network slicing model based on the purpose of data communication. The issues and challenges faced by the existing research works are reviewed and discussed in the research gaps and issues section. Moreover, the analysis carried out in this survey is based on the evaluation parameters, network layer, network parameters, system, purpose and simulation toolsets. The future work of the URLLC research is based on the issues collected from the recent research papers reviewed in this study.
\end{abstract}

Keywords: Network Slicing, Cellular Network, Medium Access Control, Wireless Communication, Mobility Management

\section{Introduction}

$5 \mathrm{G}$ technology is designed using various requirements from the industrial and social sectors, where wireless technology enables the variety of diverse use cases and applications, which in turn pave the way for business models. These requirements are highly demanding in wireless technology (Colazzo et al., 2016). The $5 \mathrm{G}$ system will not only enhance the data rate but also meet the demands for services from industrial verticals. The network services have various needs such as network performance, like communication reliability, channel utilization, throughput and latency access (Ksentini et al., 2018). 5G network encompasses fixedmobile convergence with the connectivity speed in the multi-tenancy infrastructure. Integrating the diverse technologies with single network architecture using the network functions poses technology challenges in terms of scalability, energy efficiency and security (Siddiqui et al.,
2016). However, the 5G network consists of a Heterogeneous Network (HetNet). In 5G small cells, the capacity of the network is computed to support the supermassive connection and large traffic through mmWave technology (Liang et al., 2016). 5G cellular network provides better throughput and reduced the transfer latency in the communication systems. 5G requires a better data rate in video streaming and low latency in the vehicle to vehicle transportation to ensure transportation safety (Huang et al., 2017). Network slicing is one of the key components in $5 \mathrm{G}$ wireless systems, where the major objective is to design the network architecture to support the Internet of Things (IoT) and massive Machine-Type Communications (mMTC), enhanced Mobile Broadband services (eMBB) and applications and URLLC communications. Besides the human-oriented smart devices, the 5G systems include vehicles, actuators and sensors to support more than 50 use case scenarios (Ksentini and Nikaein, 2017). 
However, network slicing is envisioned to create the network instances, which helps to share the network infrastructure and guarantees the requirements in heterogeneous networks (Ksentini et al., 2018). URLLC is envisioned to support the automotive industry services, like industry 4.0, vehicular networks, vertical industries such as smart grid, intelligent transport and smart home environment and other services, namely tactile internet and cloud robotics. All the network services need low latency access in the communication scenario (Ksentini et al., 2018). The millimeter-Wave (mmWave) communication captured significant attention in both academia and industry, as it poses large scale antennas and wide bandwidth availability. However, mmWave backhaul is one of the important technologies used in $5 \mathrm{G}$ network, which is formed by combining the HetNet with mmWave communication. It is widely used to transfer heavy traffic to the internet (Liang et al., 2016). This survey is focused on reviewing various low latency 5G communication techniques at PHY, MAC and cross-layer aspects. Categorizing the considered research papers into five groups such as network slicing, scheduling, modulation, spectrum sensing and frame structure. The paper provides a common review of trends observed in the $5 \mathrm{G}$ research considering various aspects of communication techniques used in 5G. This survey will help the researchers to get an insight into the trends observed in the various communication techniques in 5G. Also this work focusses on clearly mentioning the approach used by other researchers considered in this study and shows how it becomes beneficial in improving the latency, bandwidth utilization and increased performance in the $5 \mathrm{G}$ system.

This paper is organized as follows: Section 1 discusses the introduction and section 2 presents the motivation of the survey. Section 3 focusses on the related works while section 4 presents the research gaps and issues. Section 5 elaborates the analysis of various factors and finally, section 6 concludes the paper.

\section{Motivation}

The 5G networks render three specific key services, enhanced Mobile Broadband communication (eMBB) services, massive Machine Type Communication (mMTC) based services and Ultra Reliable Low Latency Communications (URLLC) based services. Here, various techniques, such as scheduling, network slicing, resource reuse based model and radio resource management are introduced to provide efficient communication with low latency and high reliability in PHY/MAC layers. However, the challenges associated with these techniques motivate the researchers to design a new model for $5 \mathrm{G}$ communication networks.

\section{Related Works}

This section elaborates on the communication methods for $5 \mathrm{G}$ network from various existing research papers. Moreover, the categorization of the communication techniques is represented in Fig. 1.

\begin{tabular}{|c|c|c|c|c|c|}
\hline & & $\begin{array}{c}5 \mathrm{G} \\
\text { communication } \\
\text { techniques }\end{array}$ & $\begin{array}{l}\text { ation } \\
\text { es }\end{array}$ & & \\
\hline $\begin{array}{l}\text { Structure base } \\
\text { communication } \\
\text { technique }\end{array}$ & $\begin{array}{c}\text { Resource } \\
\text { reuse based } \\
\text { technique }\end{array}$ & $\begin{array}{c}\text { Scheduling } \\
\text { based technique }\end{array}$ & $\begin{array}{l}\text { Diversity based } \\
\text { technique }\end{array}$ & $\begin{array}{c}\text { Radio resource } \\
\text { management } \\
\text { technique }\end{array}$ & $\begin{array}{c}\text { Network slicing } \\
\text { technique }\end{array}$ \\
\hline $\begin{array}{l}\text { Commonly used } \\
\text { approaches: }\end{array}$ & $\begin{array}{l}\text { Commonly } \\
\text { used } \\
\text { approaches: }\end{array}$ & $\begin{array}{l}\text { Commonly used } \\
\text { approaches: }\end{array}$ & $\begin{array}{l}\text { Commonly used } \\
\text { approaches: }\end{array}$ & $\begin{array}{l}\text { Commonly used } \\
\text { approaches: }\end{array}$ & $\begin{array}{l}\text { Commonly } \\
\text { used } \\
\text { approaches: }\end{array}$ \\
\hline $\begin{array}{l}\text { - MAC layer } \\
\text { protocol based } \\
\text { on reducing } \\
\text { latency and } \\
\text { optimum band } \\
\text { width utilization }\end{array}$ & $\begin{array}{l}\text { - Based on } \\
\text { cross layer } \\
\text { PHY and } \\
\text { MAC layer } \\
\text { protocols }\end{array}$ & $\begin{array}{l}\text { - MAC } \\
\text { scheduling } \\
\text { model } \\
\text { - Cross layer } \\
\text { resource } \\
\text { allocation model } \\
\text { - Modeling 5G } \\
\text { frame structure } \\
\text { - QoS aware } \\
\text { MAC scheduler }\end{array}$ & $\begin{array}{l}\text { - Coding and } \\
\text { modulation } \\
\text { schemes } \\
\text { - Energy } \\
\text { efficient } \\
\text { modulation } \\
\text { schemes } \\
\text { - Power aware } \\
\text { MAC protocol }\end{array}$ & $\begin{array}{l}\text { - Adaptive radio } \\
\text { link control } \\
\text { protocol } \\
\text { - Radio access } \\
\text { network based } \\
\text { architecture }\end{array}$ & $\begin{array}{l}\text { - Network } \\
\text { slicing model } \\
\text { for } 5 \mathrm{G} \\
\text { communication } \\
\text { system } \\
\text { - Network } \\
\text { slicing model } \\
\text { for radio access } \\
\text { network } \\
\text { - Network } \\
\text { slicing based } \\
\text { resource } \\
\text { management } \\
\text { model }\end{array}$ \\
\hline
\end{tabular}

Fig. 1: Categorization of 5G communication techniques 


\section{Structure-Based Communication Techniques}

The research papers based on structure-based communication techniques for $5 \mathrm{G}$ wireless communication are discussed below: (Zhang et al., 2019) developed a joint bandwidth and latency constrained grouping algorithm to generate the network unit group for various network slice environment. It effectively provided support for latency optimization and bandwidth resource. The network unit group meets the requirements of network services to ensure bandwidth utilization. Based on the requirements of latency, the pooling cycle was shortened and reduced which leads to increase the coverage or reduce the burst transmission. The network model was validated only under fiber lengths, service ratios and network loads. Alfadhli et al. (2019) developed a latency reduction model to achieve reliable latency communication in $5 \mathrm{G}$ networks. It effectively enabled the data to be transmitted instantly which reduced the delay in fronthaul. Moreover, it considered some additional functions at edge nodes such as a remote unit, which in turn reduced the latency in the communication network. Correa and David (2018) modelled a custom protocol to reduce the latency and to increase the speed of communication channels in $5 \mathrm{G}$ networks. The utilization of channel bandwidth attained better efficiency of $89 \%$ in terms of the payload packets of 100 bytes. The communication stack of this protocol remained sufficient and simple in performing the clustering operations. However, the clock cycle waste present in the controller misused the valuable time. Raviraj et al. (2005) developed a MAC layer protocol named MOBMAC for supporting mobility in sensor networks. It used the frame size model to solve the issues of frame loss caused by the Doppler shift in mobile scenarios. The frame size used to perform the data transmission was predicted using the extended Kalman filter, which in turn reduced the latencies and increased the efficiency of the system. Zheng et al.
(2010) modeled an energy-efficient and low latency MAC protocol for data transmission in a multi-hop network. It attained better energy efficiency and lower latency. Here, the routing information was used to alter the format of the controlling frame. It utilized the carrier listening technology to involve the neighboring nodes to accomplish the process of multi-hop delivery. Zafar and Khan (2017) developed a multi cluster-based communication protocol in the network management to reduce the communication cost. It optimized the network performance by exploiting low power and low complexity in the intra-cluster and inter-cluster communication. Here, both the beaconless and beacon-enabled modes were investigated using the time division multiple access. It eliminated the access delay and collision. The summary of the above discussion has been mentioned in Table 1 .

\section{Resource Reuse-based Communication Techniques}

This section elaborates on the resource reuse based 5G communication techniques gathered from various research papers. Mathur et al. (2016) introduced a crosslayer MAC and PHY layer protocol to solve the issues in bitrate limited devices. It required a wide range of data communication services and less access delay and effectively performed the operations in the same band. It allowed the IoT devices not to wait for accessing the small-sized data transmission. It reduced the control overhead, but the utilization of the underlay spectrum was considered as self-interference. Papaioannou et al. (2018) developed an optical fronthaul architecture to synergize the optical transceiver, optical multiplexer and beamforming more effectively towards the analog fronthaul in the medium transparent small cell networks. Here, the Remote Radio Heads (RRHs) offered the wireless data rates by ranging from 25 to 400 Gbps.

Table 1: Summary of structure based communication techniques

\begin{tabular}{|c|c|c|}
\hline Author & Approach & Advantages \\
\hline Zhang et al. (2019) & $\begin{array}{l}\text { Joint bandwidth and latency constrained algorithm } \\
\text { Based on requirement of latency, pooling cycle } \\
\text { was shortened and reduced. }\end{array}$ & $\begin{array}{l}\text { Latency optimization and bandwidth utilization } \\
\text { reduces the burst transmission and increases coverage }\end{array}$ \\
\hline Alfadhli et al. (2019) & Latency reduction model & Reliable latency, reduction of delay in fronthaul \\
\hline $\begin{array}{l}\text { Correa and David } \\
\text { (2018) }\end{array}$ & $\begin{array}{l}\text { modelled a protocol to reduce latency and increase } \\
\text { speed of communication }\end{array}$ & $\begin{array}{l}\text { Better efficiency of } 89 \% \text { in terms of payload packets } \\
\text { of } 100 \text { bytes. }\end{array}$ \\
\hline Raviraj et al. (2005) & Developed MAC layer protocol-MOBMAC & $\begin{array}{l}\text { Used the frame size model to solve frame loss issues in } \\
\text { wireless sensor networks using Kalman filter. Hence, } \\
\text { reduced latency and increased efficiency. }\end{array}$ \\
\hline Zheng et al. (2010) & MAC protocol for multi hop network & $\begin{array}{l}\text { Routing information was used to alter the format of } \\
\text { controlling frame. Carrier listening was used to } \\
\text { accomplish multi hop delivery. }\end{array}$ \\
\hline $\begin{array}{l}\text { Zafar and Khan } \\
(2017)\end{array}$ & Multi cluster communication protocol & $\begin{array}{l}\text { Reduces cost by exploiting low power and low } \\
\text { complexity in the intra cluster and inter cluster } \\
\text { communication. }\end{array}$ \\
\hline
\end{tabular}


However, the resource allocation and small cell access were ensured through MAC protocol. It failed to monitor access control and network operations. Chung (2019) modelled a low latency and high speed passive optical network model to solve the issues in the bandwidth and latency requirements of $5 \mathrm{G}$ wireless services. It utilized low latency operations to increase the performance of the system. It effectively realized the flexibility for adding or replacing the new functions in the network services. Liang et al. (2016) developed a MAC protocol model for mmWave in 5G wireless network. It utilized the signaling, frame structure and backhaul or downlink or uplink resource allocation scheme at the system level in backhaul network. It efficiently connected the end-users and increased network capacity. It failed to perform backhaul routing and resource allocation in the mmWave system. Labiod et al. (2019a) developed a cross-layer model for video streaming in a wireless network. It provided better coding efficiency and low delay in the coding structure. The coding structure guaranteed the time of minimal coding in the video bitrates, which penalized the performance of the transmission. To increase the quality of the received video, the cross-layer model was utilized in vehicular communications. It offered better quality enhancements at the receiver side. However, the efficiency of video encoding was significantly poor in video prediction. Labiod et al. (2019b) developed an adaptive cross-layer model for video streaming in $5 \mathrm{G}$ network. The cross-layer model increased the performance of video streaming under fewer delay constraints. The transmitted video packets were assigned to the access queue in the MAC layer by considering the prediction structure. It does not perform as a classification module in the encoding configuration. Ma et al. (2017) modelled a handover authentication mechanism based on hypothesis tests in wireless communication. The fundamental parameters were designed in the physical layer to achieve the authentication process. This mechanism consumed less storage and computational resources. This framework provided a better performance in terms of security. The alarm-rate and the detection rate were significantly poor in the authentication process. Levanen et al. (2017) developed a low latency interference model to balance the amount of memory in the physical layer process of $5 \mathrm{G}$ network. It reduced the time duration while transmitting the frames by increasing the frequency allocation. It achieved better performance in data communication. Yang et al. (2015) developed a physical layer security model in $5 \mathrm{G}$ network under the frequency spectrum. It takes advantage of data confidentiality using the security paradigm in 5G. It exploited the intrinsic randomness to achieve the benefit of data security in 5G technology. It showed better performance in MIMO system. Baig et al. (2017) developed a dual physical layer transceiver in 5G to enable the pulse shaping in signal transmission. It utilized sub-layers to ensure the efficiency of $4 \mathrm{G}$ network devices with confinement, increased robustness and higher spectral efficiency. However, it was not effective in using wavelet filter banks. Ford et al. (2017) introduced a mmWave based low latency approach in 5G wireless cellular networks. The spectrum with highfrequency regions was effectively marked and bands with $10 \mathrm{GHz}$ bandwidth were used to achieve the data communication. However, the frequency bands of mmWave support data rates and services while maintaining low latency and reliability. Levanen et al. (2014) developed a low latency interference approach in designing the $5 \mathrm{G}$ communication area. It provided the transmission latencies of $1 \mathrm{~ms}$ and increased the spectral efficiency of the system. However, the delay in the radio interface was invisible to the higher layers. Ejaz and Ibnkahla (2017) developed a multiband spectrum sensing model in the cognitive network for 5G IoT devices. The multiband model decreased the energy consumption in spectrum sensing than the single band approach. It effectively satisfies the probabilities of false alarm and detection requirements to reduce the energy by determining the number of channels in the IoT nodes. It was not suitable for mobile IoT nodes, where the channel fading alters over time. Kim et al. (2014) developed a physical layer-based MAC framework to increase the throughput of data traffic in a sensor network.

Table 2: Summary of resource reuse based communication techniques

\begin{tabular}{|c|c|c|}
\hline Author & Approach & Advantages \\
\hline Mathur et al. (2016) & Cross layer MAC and PHY layer protocol & Solved the issues in bitrate limited devices \\
\hline $\begin{array}{l}\text { Papaioannou et al. } \\
\text { (2018) }\end{array}$ & $\begin{array}{l}\text { Developed optical fronthaul architecture to synergize the } \\
\text { optical transceiver, optical multiplexer and beamforming } \\
\text { more effectively towards the analog fronthaul in the medium } \\
\text { transparent small cell networks }\end{array}$ & $\begin{array}{l}\text { Resource allocation and small cell access } \\
\text { were ensured through MAC protocol }\end{array}$ \\
\hline Chung (2019) & Developed low latency, high speed passive optical network & Solved issues in bandwidth and latency \\
\hline Liang et al. (2016) & Developed low latency, high speed passive optical network & Increased network capacity. \\
\hline Labiod et al. (2019a) & $\begin{array}{l}\text { Developed a cross layer model for video streaming in } \\
\text { wireless network }\end{array}$ & Used in vehicular communication \\
\hline Levanen et al. (2017) & $\begin{array}{l}\text { Developed low latency interference model to balance the amount } \\
\text { of memory in the physical layer process of } 5 \mathrm{G} \text { network. }\end{array}$ & $\begin{array}{l}\text { Achieved better performance in } \\
\text { data communication }\end{array}$ \\
\hline Yang et al. (2015) & $\begin{array}{l}\text { Developed a PHY layer security model in } 5 \mathrm{G} \text { network } \\
\text { under the frequency spectrum. }\end{array}$ & $\begin{array}{l}\text { Showed better performance in MIMO } \\
\text { system. }\end{array}$ \\
\hline
\end{tabular}


Here, the acknowledgments were parallelized from sensor nodes to pull messages. The access point node knows which node has the data to forward and to allocate the channel resources by transmitting the pull message. It failed to address the strategy of group scheduling and grouping in the probe and pull mechanism. Biswash and Jayakody (2018) developed a user-centric dynamic switching and mobility management approach to increase the performance in data communication. It acts as the central node in the user mobility and switching mode during communication. However, the mode switching was based on the parameters, like delay, energy consumption and link utilization. It increased the performance of mobility management and achieved better scalability and robustness. It does not guarantee the quality of the network. The summary of the above discussion has been mentioned in Table 2.

\section{Scheduling based Techniques}

The research papers that utilized the scheduling based $5 \mathrm{G}$ communication techniques in URLLC are explained in this section. Ksentini et al. (2018) developed a two-level MAC scheduling model to effectively handle the downlink and uplink transmission in the network slices with various characteristics. The transmission scheduling was efficiently computed at the radio access network level. It offered more flexibility in managing the radio resources to meet the requirements of latency in communication services. However, the burden in the scheduling process at MAC layer was not resolved. Huang et al. (2017) developed a MAC scheduling model in the massive MIMO system. It enabled the processing parallelization. Scheduling in the resource blocks was executed concurrently based on the common post and pre-processing. The power-based weight is included in the scheduling to complete the link adaptation. It failed to increase throughput and transmitting power. Wang et al. (2019) developed a cross-layer resource allocation model for optimizing the rate control in the long term perspective of the wireless network. It effectively decreased the delay and increased the reliability of the network based on the relay model. It separated the crosslayer optimization problem into power allocation and rate control problem. The problem was solved using the alternating direction scheme. It failed to combine the big data with the alternating direction model to solve the complexity issues. Lähetkangas et al. (2014) modelled a $5 \mathrm{G}$ frame structure to initiate the data transmission or reception time, like scheduling, signaling and synchronization. With the same battery and power consumption, the frame structure reduced the energy consumption in the network design. However, utilizing the subcarrier spacing and data parts in the frame was not considered. Zhang et al. (2014) developed an energyefficient low latency model in 5G network. It utilized the top-down approach for designing the low latency and energy-efficient reliable networks. It effectively considered the air interface and user-centric scheduling to make the network as energy efficient. Pocovi et al. (2017) developed a low latency communication framework in the cellular networks. It used the low pass interference information to find the imperfection in link adaptation in sporadic traffic. It achieved a strong correlation between channel quality and user latency. However, the scheduling process and the link adaptation mechanism were not considered in the mixed traffic class. Chaudhuri et al. (2018) introduced a QoS aware MAC scheduler to adhere to the fairness and quality requirements in the network specification. Here, the multi-objective optimization was used to select the right user and their corresponding application to meet the requirements of quality. It attained better cell throughput and minimum packet drop rate in the MAC scheduler. It failed to increase the performance of video streaming users. The summary of the above discussion has been mentioned in Table 3.

Table 3: Summary of Scheduling based communication techniques

\begin{tabular}{|c|c|c|}
\hline Author & Approach & Advantages \\
\hline $\begin{array}{l}\text { Huang et al. } \\
\text { (2017) }\end{array}$ & $\begin{array}{l}\text { Developed a MAC scheduling model in the } \\
\text { massive MIMO system }\end{array}$ & $\begin{array}{l}\text { Scheduling in the resource blocks was executed concurrently based } \\
\text { on the common post and pre-processing. The power-based weight } \\
\text { is included in the scheduling to complete the link adaptation. }\end{array}$ \\
\hline Wang et al. (2019) & $\begin{array}{l}\text { Developed a cross-layer resource allocation } \\
\text { model for optimizing the rate control in the } \\
\text { long term perspective of the wireless network }\end{array}$ & $\begin{array}{l}\text { Effectively decreased the delay and increased the } \\
\text { reliability of the network based on the relay model }\end{array}$ \\
\hline $\begin{array}{l}\text { Lähetkangas et al. } \\
\text { (2014) }\end{array}$ & $\begin{array}{l}\text { Modelled a } 5 \mathrm{G} \text { frame structure to initiate the } \\
\text { data transmission or reception time, like } \\
\text { scheduling, signaling and synchronization }\end{array}$ & $\begin{array}{l}\text { Frame structure reduced the energy consumption in the } \\
\text { network design within the same battery and power } \\
\text { consumption, }\end{array}$ \\
\hline Zhang et al. (2014) & $\begin{array}{l}\text { Developed an energy-efficient low latency } \\
\text { model in } 5 \mathrm{G} \text { network }\end{array}$ & $\begin{array}{l}\text { Utilized the top-down approach for designing the low } \\
\text { latency and energy-efficient reliable networks. }\end{array}$ \\
\hline $\begin{array}{l}\text { Pocovi et al. } \\
(2017)\end{array}$ & $\begin{array}{l}\text { Developed low latency communication } \\
\text { framework in the cellular networks. }\end{array}$ & $\begin{array}{l}\text { Utilized the low pass interference information to find the } \\
\text { imperfection in link adaptation in sporadic traffic. It achieved } \\
\text { a strong correlation between channel quality and user latency }\end{array}$ \\
\hline $\begin{array}{l}\text { Chaudhuri et al. } \\
\text { (2018) }\end{array}$ & $\begin{array}{l}\text { Developed a QoS aware MAC scheduler to } \\
\text { adhere to the fairness and quality } \\
\text { requirements in the network specification }\end{array}$ & $\begin{array}{l}\text { It attained better cell throughput and minimum packet drop } \\
\text { rate in the MAC scheduler }\end{array}$ \\
\hline
\end{tabular}


Table 4: Summary of diversity based techniques

\begin{tabular}{|c|c|c|}
\hline Author & Approach & Advantages \\
\hline Sachs et al. (2018a) & $\begin{array}{l}\text { Introduced a coding and modulation scheme } \\
\text { for increasing the reliability for URLLC networks. }\end{array}$ & Increasing the reliability for URLLC networks \\
\hline Khalid et al. (2018) & $\begin{array}{l}\text { Developed an energy-efficient modulation } \\
\text { mechanism in the } 5 \mathrm{G} \text { femtocell network to reduce } \\
\text { the consumption of energy in data transmission }\end{array}$ & $\begin{array}{l}\text { Saves energy and reduces data usage by a } \\
\text { thousand-fold in } 5 \mathrm{G}\end{array}$ \\
\hline $\begin{array}{l}\text { Franchino and Buttazzo } \\
\text { (2017) }\end{array}$ & $\begin{array}{l}\text { Power-aware MAC layer protocol to enhance the } \\
\text { lifetime of the system }\end{array}$ & $\begin{array}{l}\text { Effectively allocated and shared the bandwidth } \\
\text { to all the nodes in the wireless network. }\end{array}$ \\
\hline Hoang et al. (2014) & $\begin{array}{l}\text { Developed a cooperative MAC protocol for bi- } \\
\text { directional traffic based on the network coding } \\
\text { mode }\end{array}$ & $\begin{array}{l}\text { It increased the performance of the network } \\
\text { through a cross-layer perspective in terms of the } \\
\text { parameters such as end-to-end latency and } \\
\text { network throughput. }\end{array}$ \\
\hline Sayadi et al. (2018) & $\begin{array}{l}\text { Introduced a radio over Ethernet protocol for } \\
\text { data transmission at the Ethernet frame in } 5 \mathrm{G} \\
\text { fronthaul network }\end{array}$ & Increased performance \\
\hline
\end{tabular}

\section{Diversity-based Techniques}

This section elaborates on the diversity-based communication techniques for $5 \mathrm{G}$ wireless networks. Sachs et al. (2018a) introduced a coding and modulation scheme for increasing the reliability for URLLC networks. It was specified that low latency features will minimize transmission latency. However, the redundancy was provided over various transmission points and carriers with the carrier aggregation model. Khalid et al. (2018) developed an energy-efficient modulation mechanism in the $5 \mathrm{G}$ femtocell network to reduce the consumption of energy in data transmission. The adaptive modulation saves energy and reduces data usage by a thousand-fold in 5G. Franchino and Buttazzo (2017) introduced a power-aware MAC layer protocol to enhance the lifetime of the system by bounding the delay of real-time messages. It effectively allocated and shared the bandwidth to all the nodes in the wireless network. The protocol performed multihop communication in various network topologies. However, it failed to perform the delay analysis in case of downstream flow. Hoang et al. (2014) developed a cooperative MAC protocol for bi-directional traffic based on the network coding mode. It increased the performance of the network through a cross-layer perspective in terms of the parameters such as end-toend latency and network throughput. The performance was validated using Monte-Carlo simulation. It failed to integrate the network layer with the cross-layer in a wireless network. Sayadi et al. (2018) introduced a radio over Ethernet protocol for data transmission at the Ethernet frame in 5G fronthaul network. Here, the mapper present at the transmitter side received the data and forward it to the Ethernet frame. The mapper situated at the destination end received the Ethernet frame and extract the data. However, running the mapper in the embedded system failed to reduce the delay in data transmission. The summary of the above discussion has been mentioned in Table 4 .

\section{Radio Resource Management Methods}

This section elaborates on the radio resource management techniques in $5 \mathrm{G}$ wireless network collected from the existing research papers. Roth-Mandutz et al. (2017) developed a self-optimized model at the network management level for reducing the capacity impact in ultra-reliable communication services. It effectively selected the appropriate parameters and identified the legacy use cases. It enhanced the performance by meeting the diverse requirements. However, it failed to enhance the parameter optimization at the user-centric and service network. Colazzo et al. (2016) developed a Novel Radio Multiservice Adaptive network model (NORMA) in the 5G network to achieve low latency wireless communication. Here, the network slice effectively allocated the network functions to be required in the radio interface. Based on the lean protocol stack, the packets were able to pass over the stack layers more efficiently. Dighriri et al. (2016) developed a data traffic model in $5 \mathrm{G}$ network to achieve machine to machine communication. Based on the measure and classification of data traffic, the quality of service in the network slicing was effectively satisfied in smart systems. It efficiently utilized the radio resources in the relay node of the physical resource block. The fairness of service and the data traffic explosion was not resolved. Rony et al. (2018) introduced a radio access network based architecture in $5 \mathrm{G}$ network. The functionality split at the physical layer effectively tackled the challenges associated with the radio access network. It supports the centralization of the upper layers to achieve a high data rate. It was not effective in traffic load conditions. Shreevastav and Carbajo (2016) developed an adaptive radio link control protocol to increase the throughput of network modalities in a cellular system. It reduced the processing power and increased the system latency by enabling the un-acknowledgment mode. However, the radio condition activates the acknowledgment mode to increase data reliability. It was not suitable in 5G split architectural 
environment. The summary of the above discussion has been mentioned in Table 5 .

\section{Network Slicing Techniques}

Some of the network slicing methods collected from the existing research papers are represented in this section. Li et al. (2016) introduced a network slicing model for $5 \mathrm{G}$ communication systems. This model was based on the end to end horizontal and vertical slicing, where horizontal slicing increases the user experience and system capacity whereas vertical slicing enables the vertical services. However, the communication and the computation resources were horizontally sliced to generate the virtual computation environment to solve the network scaling problems. However, the granularity and the ways of doing network slicing were not primarily focused. Ksentini and Nikaein (2017) developed a network slicing model in the radio access network. This model relies on the principle of a dedicated core network to separate the traffic and to share the resources between the slices. It enabled the dynamicity and flexibility to enforce the network slicing in the radio access network. However, the two-level scheduling processes for the stability, performance and scalability were not addressed. Rost et al. (2017) developed a network slicing model to design the $5 \mathrm{G}$ mobile networks. It effectively analyzed the realization options in the 5G network based on the network slices. It offered revenue to the network operator and achieved mapping with the shared and the dedicated slices. Siddiqui et al. (2016) introduced virtualized intelligent and hierarchical network architecture. It explored the hierarchical routing in the network caching to achieve the requirements of low latency. It effectively solved the issues of multi-tenancy and security in the network slicing. It failed to reduce the content-based access latency in fixed and mobile networks. Herzog et al. (2016) developed a network slicing paradigm to enable agile, scalable and highly flexible 5G compatible network architecture.MAC layer can support the multi-radio access operations to maximize the traffic volume, which effectively enhanced the quality of different users in the network system. However, partitioning and managing the resources across the heterogeneous networks was not achieved. Chen et al. (2019) developed a network slicing based resource management model to modify the control model of network architecture. For event and application types, the network resources offered suitable prioritization and bandwidth and ensure the reliability of data transmission. It failed to consider the stability of the system even under abnormal events. The summary of the above discussion has been mentioned in Table 6 .

Table 5: Summary of Radio resource management based communication techniques

\begin{tabular}{|c|c|c|}
\hline Author & Approach & advantages \\
\hline Colazzo et al. (2016) & $\begin{array}{l}\text { Developed a novel radio multiservice adaptive } \\
\text { network model (NORMA) in the } 5 \mathrm{G} \text { network }\end{array}$ & Low latency wireless communication \\
\hline Dighriri et al. (2016) & Developed a data traffic model in $5 \mathrm{G}$ network & $\begin{array}{l}\text { It efficiently utilized the radio resources in the } \\
\text { relay node of the physical resource block }\end{array}$ \\
\hline Rony et al. (2018) & $\begin{array}{l}\text { Introduced a radio access network based } \\
\text { architecture in } 5 \mathrm{G} \text { network }\end{array}$ & $\begin{array}{l}\text { Tackled the challenges associated with the radio } \\
\text { access network }\end{array}$ \\
\hline $\begin{array}{l}\text { Shreevastav and Carbajo } \\
\text { (2016) }\end{array}$ & $\begin{array}{l}\text { Developed an adaptive radio link control } \\
\text { protocol }\end{array}$ & $\begin{array}{l}\text { Increase the throughput of network modalities in } \\
\text { a cellular system }\end{array}$ \\
\hline
\end{tabular}

Table 6: Summary of Network Slicing techniques

\begin{tabular}{|c|c|c|}
\hline Author & Approach & advantages \\
\hline Li et al. (2016) & $\begin{array}{l}\text { Introduced network slicing model for } 5 \mathrm{G} \\
\text { communication systems. }\end{array}$ & Increases the user experience and system capacity \\
\hline $\begin{array}{l}\text { Ksentini and Nikaein } \\
(2017)\end{array}$ & $\begin{array}{l}\text { Developed network slicing model in the } \\
\text { radio access network. }\end{array}$ & $\begin{array}{l}\text { It enabled the dynamicity and flexibility to enforce the } \\
\text { network slicing in the radio access network }\end{array}$ \\
\hline Rost et al. (2017) & Developed a network slicing model & $\begin{array}{l}\text { It effectively analysed the realization options in the } 5 \mathrm{G} \\
\text { network based on the network slices }\end{array}$ \\
\hline Siddiqui et al. (2016) & $\begin{array}{l}\text { Introduced virtualized intelligent and } \\
\text { hierarchical network architecture. }\end{array}$ & Achieved the requirements of low latency \\
\hline Herzog et al. (2016) & $\begin{array}{l}\text { Developed a network slicing paradigm to } \\
\text { enable agile, scalable and highly flexible } \\
5 \mathrm{G} \text { compatible network architecture }\end{array}$ & $\begin{array}{l}\text { Enhanced the quality of different users in the network } \\
\text { system }\end{array}$ \\
\hline Chen et al. (2019) & $\begin{array}{l}\text { Developed a network slicing based resource } \\
\text { management model to modify the control } \\
\text { model of network architecture }\end{array}$ & $\begin{array}{l}\text { Offered suitable prioritization and bandwidth and ensure } \\
\text { the reliability of data transmission }\end{array}$ \\
\hline
\end{tabular}




\section{Other 5G Communication Techniques}

This section addresses the low latency communication methods in $5 \mathrm{G}$ network using various communication methods. Sachs et al. (2018b) developed a $5 \mathrm{G}$ low latency communication in the tactile network services. It analyzed the costs to provide low latency and reliable wireless transmission for the coverage and spectral efficiency. However, the $5 \mathrm{G}$ functionality for URLLC services was effectively achieved in the data transmission. Chung et al. (2017) developed a highspeed optical access framework in the $5 \mathrm{G}$ wireless communication networks. The data signals generated through the baseband modulators were converted into the digital form. The data rate achieved for the user experience was exceeded to $1 \mathrm{Gbps}$ and attained a better peak data rate for each user as $1.5 \mathrm{Gbps}$. Akyildiz et al. (2018) introduced flexible network architecture in the 5G wireless system for quality-aware communication. It was characterized by various innovative features, like cellular network concepts and universal network devices. It provided highly efficient communication in the heterogeneous network. Here, the external network was brought closer to the user which in turn reduced the latency. Moreover, the legacy devices were integrated with the hardware-specific unit to effectively make use of the infrastructure resources. The network capability was not effectively synchronized. Santoyo-González and Cervelló-Pastor (2018) developed a heuristic-based model to solve the linear programming issues in the $5 \mathrm{G}$ network. It defines the mathematical formulation at the network edge or fog nodes in the placement of service infrastructure. It effectively solved the optimization issues based on the position of traffic generators. The placement parameters like location cost and candidate sites were not considered. Moreira et al. (2018) developed a cross-layer authentication protocol to solve the issues in weak security and latency. The radio trusted database aims to increase the authentication of radio devices present in the wireless network. However, the entire coverage area is partitioned into various trusted zones, where each zone consists of numerous cells to initiate the authentication request. It reduced the computational complexity and minimized the recurrence of authentication. It failed to classify the profiles of HetNet to design efficient and reliable trusted zones. Al-Raweshidy et al. (2017) introduced a new intelligent model for optimally selecting the parameter sets in MAC layer. However, the optimized parameters considerably attained higher throughput. Here, the network parameters were neither validated nor simulated in the real-time scenario.

\section{Research Gaps and Issues}

The research gaps and issues faced by the considered research papers are elaborated in this survey. The summary of the research gaps and issues has been described in Table 7 .

Table 7: Summary of research gaps and issues

\begin{tabular}{|c|c|}
\hline & Issues and research gaps \\
\hline $\begin{array}{l}\text { 1. Structure based } \\
\text { Communication methods }\end{array}$ & $\begin{array}{l}\text { - Network model was validated only under the fiber lengths, service ratios and network loads } \\
\text { in (Zhang et al., 2019) } \\
\text { - Correa and David (2018) the clock cycle waste present in the controller misuse the valuable } \\
\text { time }\end{array}$ \\
\hline $\begin{array}{l}2 . \text { Resource reuse } \\
\text { based techniques }\end{array}$ & $\begin{array}{l}\text { - Ksentini et al. (2018), the utilization of the underlay spectrum was self-interference and the } \\
\text { access control and network operations were not effectively monitored in (Papaioannou } \text { et al., 2018). } \\
\text { - It failed to perform backhaul routing and resource allocation in the mmWave system in } \\
\text { (Liang et al., 2016). } \\
\text { - The efficiency of video encoding was significantly poor in video prediction in (Labiod et al., } \\
\text { 2019a). However, the classification module was not performed in the encoding configuration. } \\
\text { The Alarm rate and the detection rate were significantly poor in the authentication process } \\
\text { (Ma et al., 2017) and the resource model in (Yang et al., 2015) was not effective in using the } \\
\text { wavelet filter banks. } \\
\text { - The channel fading altering with time was not suitable for mobile IoT nodes (Ejaz and Ibnkahla, } \\
\text { 2017). It failed to address the strategy of group scheduling and grouping in the probe and pull } \\
\text { mechanism (Kim } \text { et al., 2014). } \\
\text { - The quality of the network was not guaranteed in (Biswash and Jayakody, 2018). }\end{array}$ \\
\hline $\begin{array}{l}\text { 3. Scheduling based } \\
\text { techniques }\end{array}$ & $\begin{array}{l}\text { - The burden in the scheduling process at MAC layer was not resolved in (Zhang et al., 2014) } \\
\text { and the throughput and transmitting power is reduced in (Huang et al., 2017). } \\
\text { - It failed to combine the big data with the alternating direction model to solve the complexity } \\
\text { issues according to (Wang et al., 2019). } \\
\text { - Lähetkangas et al. (2014), the subcarrier spacing and data parts are failed to use in the frame. } \\
\text { - Siddiqui et al. (2016), the performance was poor for video streaming users. }\end{array}$ \\
\hline
\end{tabular}


Table 7: Continuo

4. Diversity-based $5 \mathrm{G}$
communication techniques

5. Radio resource

management techniques

6. Network slicing techniques
- The redundancy was provided over various transmission points and carriers with the carrier aggregation model in (Sachs et al., 2018a)

- The transmission band system was not effectively examined in (Khalid et al., 2018).

- The delay analysis failed to perform in case of downstream flow (Franchino and Buttazzo, 2017).

- According to (Hoang et al., 2014), the integration of the network layer with the cross-layer in wireless networks failed.

- The mapper in the embedded system failed to reduce the delay in data transmission (Sayadi et al., 2018).

- It failed to enhance the parameter optimization at the user-centric and service network (Roth-Mandutz et al., 2017).

- The fairness of service and the data traffic explosion was not resolved in (Dighriri et al., 2016) and the resource model in (Rony et al., 2018) was not effective in traffic load condition. latency in fixed and mobile networks (Siddiqui et al., 2016). Partitioning and managing the resources across the heterogeneous networks was not achieved in (Herzog et al., 2016) and the stability of the system was not considered under abnormal events (Chen et al., 2019). The network capability

- The granularity and the ways of doing network slicing were not primarily focused in (Li et al., 2016).

- The two-level scheduling processes concerning the stability, performance and scalability were not focused (Ksentini and Nikaein, 2017).

- It failed to reduce content-based access was not effectively synchronized in (Akyildiz et al., 2018) and the placement parameters, like location cost and candidate sites, were not considered in (Santoyo-González and Cervelló-Pastor, 2018).

- The network parameters were not validated and simulated in the real-time scenario (Al-Raweshidy et al., 2017).

\section{Analysis and Discussion}

The analysis and discussion made using the existing $5 \mathrm{G}$ communication methods are elaborated in this section. However, the analysis is carried out based on the categorization of research work, network parameters, network layer, evaluation parameters, purpose, simulation tools and systems.

\section{Analysis Based on the Network}

The analysis carried out based on the category of the network is discussed in this section. Most of the papers discussed here consider $5 \mathrm{G}$ wireless networks. Four research papers used 5G mobile networks whereas the radio access network is used by one research paper. However, 5G cellular networks, 5G Hetnet, Flying Ad hoc Networks (FANET) and Software Defined Network $(\mathrm{SDN})$ are used by a single research paper. The vehicular ad hoc networks (VANET) are used by two research papers and so on. The analysis based on the network is represented in Fig. 2.

\section{Analysis based on Purpose in 5G Communication}

The analysis carried out based on the purpose in $5 \mathrm{G}$ network communication is discussed in this section. Here, network communication is widely used in eight research papers and the purpose of network slicing is used in four research works. Authentication, video streaming, beamforming and scheduling are preferred in three research papers. From Fig. 3, it is depicted that network communication is widely preferred in most of the research works.

\section{Analysis based on Simulation Tool Used}

The analysis carried out based on the simulation tool used is discussed in this section. Here, the MATLAB and the Network Simulator 2 (NS-2) is the widely used simulation tools. Moreover, the vehicle simulator, OPNET network simulator and Automated Validation of Internet Security Protocols and Applications (AVISPA) tools are used in two research papers and the NS 3 simulator is used in three research paper. From Fig. 4, it is clearly shown that MATLAB is a widely preferred tool in 5G communication URLLC research. However, adding NS 2 and NS 3 outperforms MATLAB.

\section{Analysis based on the System}

In this section, the analysis performed based on the system is discussed. Here, three research papers used the mmWave and MIMO systems. IoT and the wireless systems are used in two papers. Embedded systems, multi-user cellular systems and the mobility management system are used in single research work. Figure 5 portrays the analysis based on the system.

\section{Analysis based on Communication Techniques}

This section describes the analysis performed based on the categorization of existing methods. The resource reuse-based techniques are used in fifteen research papers and scheduling based techniques are used in seven works. Network slicing model, structure-based and other techniques are used in six research works. From Fig. 6, it is depicted that the resource reuse method is widely preferred. 


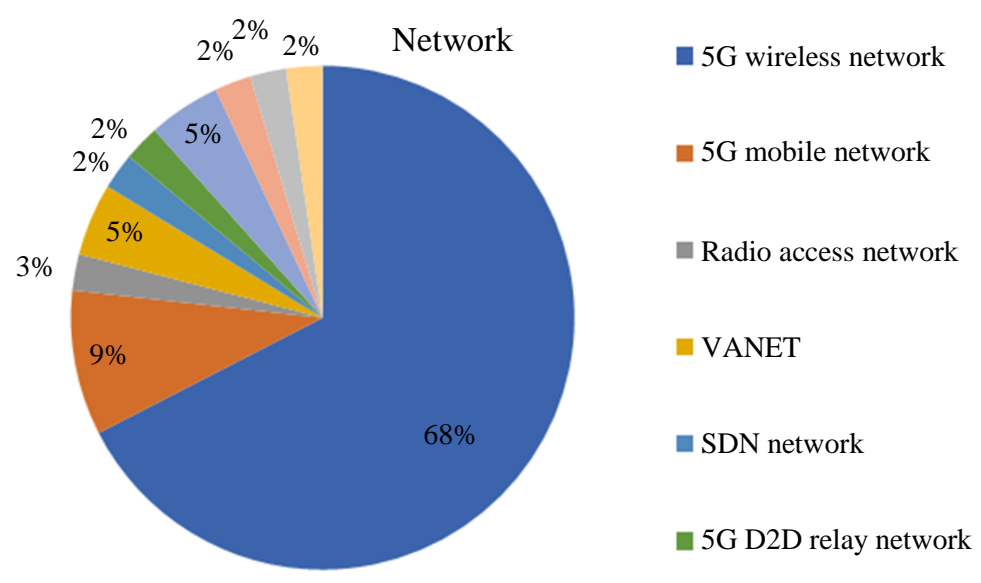

Fig. 2: Analysis based on the network

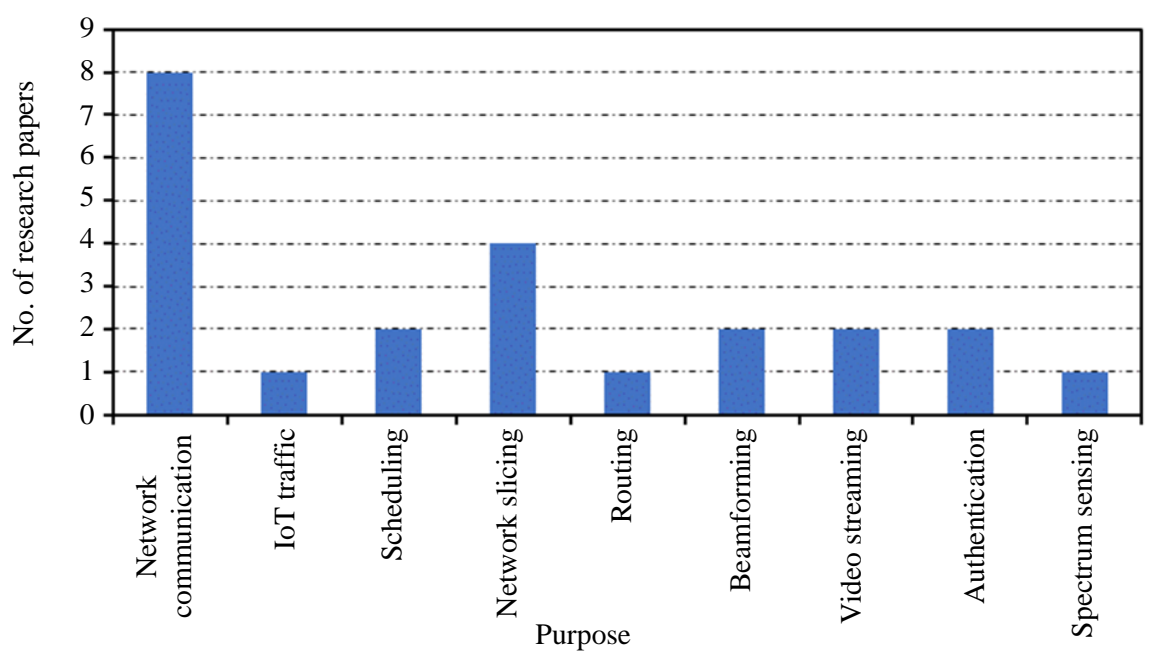

Fig. 3: Analysis based on the purpose in $5 \mathrm{G}$ communication

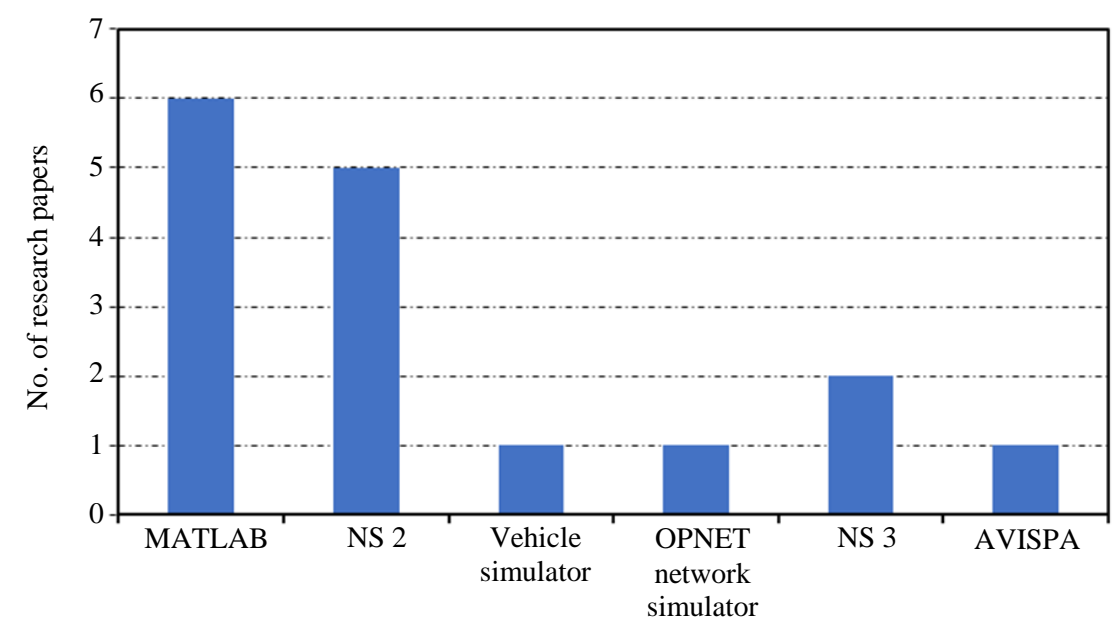

Simulation tool

Fig. 4: Analysis based on the simulation tool 
Prinu Chacko Philip and Mohammed Abdel-Hafez / Journal of Computer Science 2020, 16 (8): 1100.1114 DOI: $10.3844 /$ jessp.2020.1100.1114

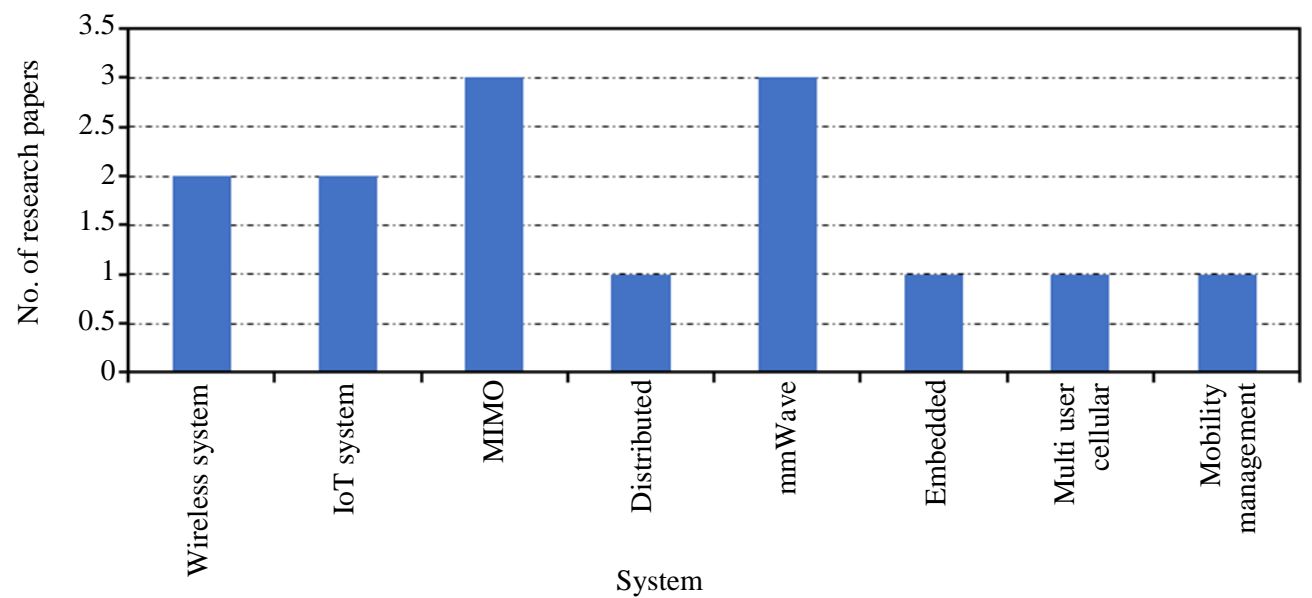

Fig. 5: Analysis based on system

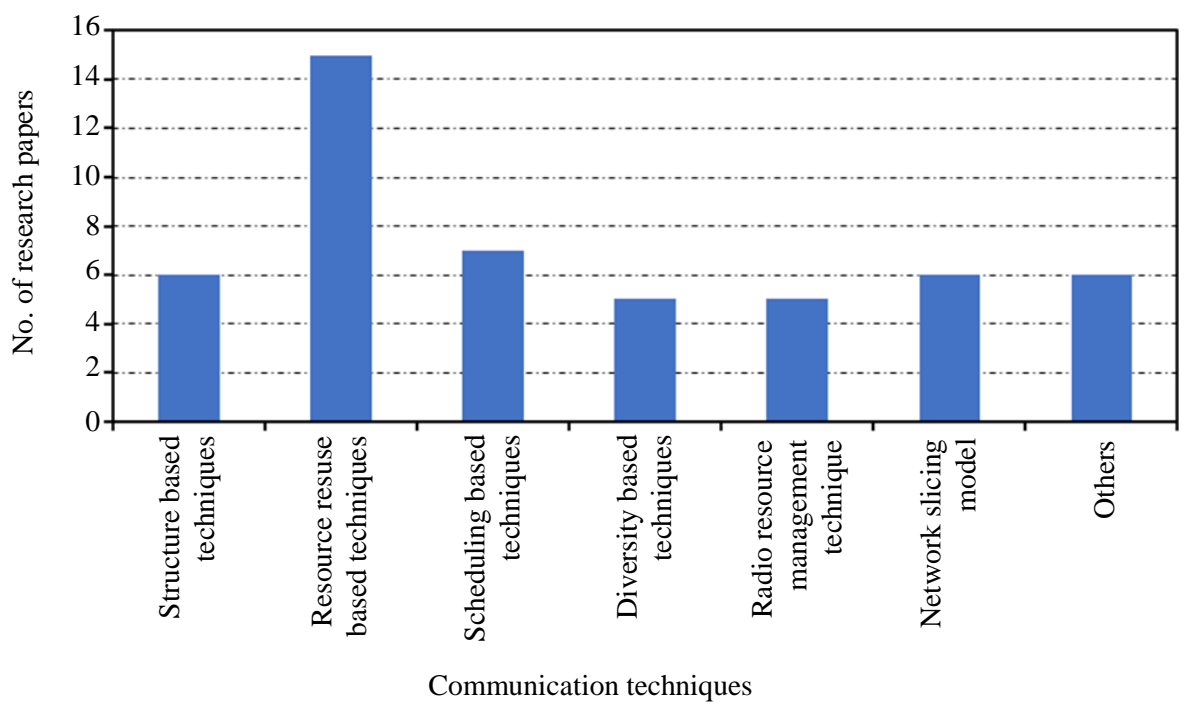

Fig. 6: Analysis based on the communication techniques

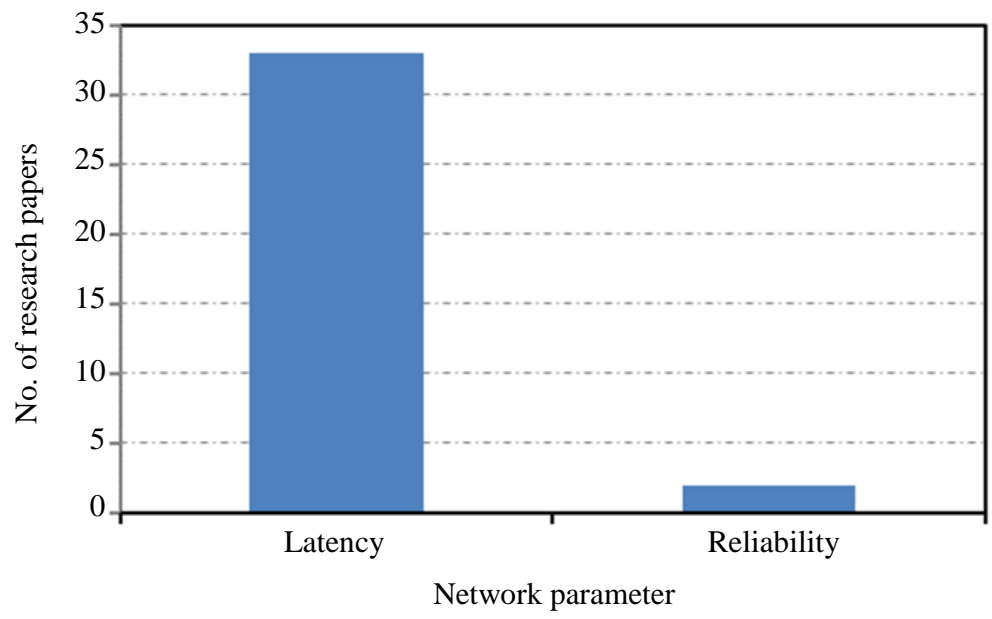

Fig. 7: Analysis based on network parameter 


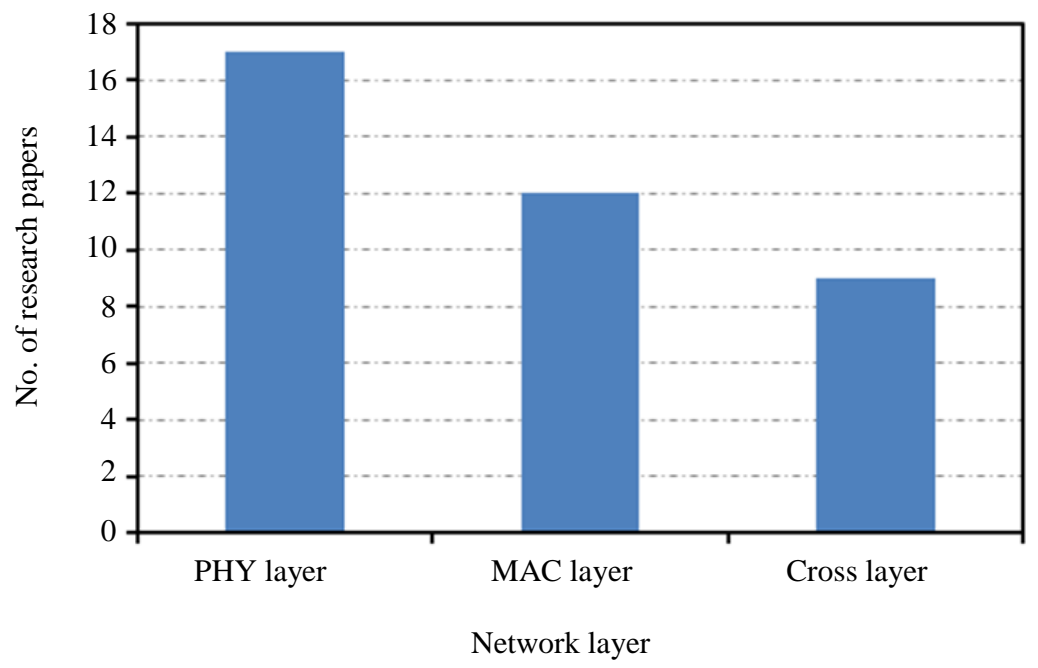

Fig. 8: Analysis based on network layer

\section{Analysis Based on Network Parameter}

This section elaborates the analysis carried out based on the network parameter in $5 \mathrm{G}$ wireless communication.

Here, the network parameters, like latency and reliability are focused and the existing research papers are categorized based on the network parameters. Here, latency is used in 33 research papers, while reliability is used in two research works. Figure 7 depicts the analysis based on network parameter.

\section{Analysis Based on Network Layer}

This section elaborates the analysis based on the network layer. Here, the PHY layer is used in 17 research papers, MAC layer is used in 12 research works and cross layer is used in nine research papers. It is clearly pictured that PHY layer is used in most of the research papers. Figure 8 portrays the analysis based on network layer.

\section{Conclusion}

The review in overall shows a general trend of the current research happenings. All the survey conclusions are based on the considered research papers. We found that $68 \%$ of research work was focused on $5 \mathrm{G}$ networks whereas the second highest of $9 \%$ research work was carried out in the $5 \mathrm{G}$ mobile wireless networks. Considering the purpose of communication in $5 \mathrm{G}$, we found that the maximum research papers were focused on network communication. Most widely used simulation tools within these research papers were the MATLAB and the network simulator 2 (NS-2). Considering the analysis based on system, mmWave and MIMO systems were found to be considered maximum in this survey. Considering the analysis based on communication technique, we found that 15 research papers considered resource reuse based technique whereas scheduling techniques were used second most.

\section{Author's Contributions}

Prinu Chacko Philip: Contributed to the writing of the manuscript and the data-analysis.

Mohammed Abdel-Hafez: Conceived the idea of review article on URLLC, contributed to the structure of the article, provided critical feedback, helped shaping the article and helped in the final version of the manuscript.

\section{Ethics}

This article is original and contains unpublished material. The corresponding author confirms that all of the other authors have read and approved the manuscript. No ethical issues were involved and the authors have no conflict of interest to disclose.

\section{References}

Akyildiz, I. F., Kak, A., Khorov, E., Krasilov, A., \& Kureev, A. (2018). ARBAT: A flexible network architecture for QoE-aware communications in $5 \mathrm{G}$ systems. Computer Networks, 147, 262-279.

Alfadhli, Y., Chen, Y. W., Liu, S., Shen, S., Yao, S., Guidotti, D., ... \& Chang, G. K. (2019). Latency performance analysis of low layers function split for URLLC applications in 5G networks. Computer Networks, 162, 106865.

Al-Raweshidy, H., Al-Kaseem, B. R., Al-Dunainawi, Y., \& Banitsas, K. (2017). A New Intelligent Approach for Optimising 6LoWPAN MAC Layer Parameters. 
Baig, S., Ahmad, M., Asif, H. M., Shehzad, M. N., \& Jaffery, M. H. (2017). Dual PHY layer for nonorthogonal multiple access transceiver in $5 \mathrm{G}$ networks. IEEE Access, 6, 3130-3139.

Biswash, S. K., \& Jayakody, D. N. K. (2018). Performance based user-centric dynamic mode switching and mobility management scheme for $5 \mathrm{G}$ networks. Journal of Network and Computer Applications, 116, 24-34.

Chaudhuri, S., Baig, I., \& Das, D. (2018). A novel QoS aware medium access control scheduler for LTEadvanced network. Computer Networks, 135, 1-14.

Chen, J. J., Tsai, M. H., Zhao, L., Chang, W. C., Lin, Y. H., Zhou, Q., ... \& Cai, Y. Z. (2019, August). Realizing Dynamic Network Slice Resource Management based on SDN networks. In 2019 International Conference on Intelligent Computing and its Emerging Applications (ICEA) (pp. 120-125). IEEE.

Chung, H. S. (2019, July). TuB2. 3-High Speed and Low Latency PON for 5G Networks. In 2019 IEEE Photonics Society Summer Topical Meeting Series (SUM) (pp. 1-2). IEEE.

Chung, H. S., Lee, H. H., Doo, K. H., Kim, K., Kim, S. H., Cho, S. H., ... \& Lee, J. H. (2017, July). Optical access technologies for $5 \mathrm{G}$ mobile communication networks. In 2017 IEEE Photonics Society Summer Topical Meeting Series (SUM) (pp. 39-40). IEEE.

Colazzo, A., Ferrari, R., \& Lambiase, R. (2016, June). Achieving low-latency communication in future wireless networks: the 5G NORMA approach. In Euro. Conf. Networks and Commun (pp. 1-5).

Correa, R. S., \& David, J. P. (2018). Ultra-low latency communication channels for FPGA-based HPC cluster. Integration, 63, 41-55.

Dighriri, M., Alfoudi, A. S. D., Lee, G. M., \& Baker, T. (2016, August). Data traffic model in machine to machine communications over $5 \mathrm{G}$ network slicing. In 2016 9th International Conference on Developments in eSystems Engineering (DeSE) (pp. 239-244). IEEE.

Ejaz, W., \& Ibnkahla, M. (2017). Multiband spectrum sensing and resource allocation for IoT in cognitive $5 \mathrm{G}$ networks. IEEE Internet of Things Journal, 5(1), 150-163.

Ford, R., Zhang, M., Mezzavilla, M., Dutta, S., Rangan, S., \& Zorzi, M. (2017). Achieving ultra-low latency in $5 \mathrm{G}$ millimeter wave cellular networks. IEEE Communications Magazine, 55(3), 196-203.

Franchino, G., \& Buttazzo, G. (2017). A power-aware MAC layer protocol for real-time communication in wireless embedded systems. Journal of Network and Computer Applications, 82, 21-34.
Herzog, U., Georgakopoulos, A., Belikaidis, I. P., Fitch, M., Briggs, K., Diaz, S., ... \& Demestichas, P. (2016). Quality of service provision and capacity expansion through extended-DSA for $5 \mathrm{G}$. Transactions on Emerging Telecommunications Technologies, 27(9), 1250-1261.

Hoang, Q. T., Nam, T. X., \& Nguyen, L. T. (2014, October). Cross-layer design of bidirectionaltraffic supported cooperative MAC protocol. In 2014 International Conference on Advanced Technologies for Communications (ATC 2014) (pp. 586-593). IEEE.

Huang, M., Yuan, F., Cheng, H., \& Zhang, X. (2017, May). Design of low-latency uplink MAC scheduling for Massive MIMO-OFDM systems. In 2017 IEEE International Conference on Communications Workshops (ICC Workshops) (pp. 632-638). IEEE.

Khalid, N., Yilmaz, T., \& Akan, O. B. (2018). Energy-efficient modulation and physical layer design for low terahertz band communication channel in $5 \mathrm{G}$ femtocell Internet of Things. Ad Hoc Networks, 79, 63-71.

Kim, T., Love, D. J., Skoglund, M., \& Jin, Z. Y. (2014). An approach to sensor network throughput enhancement by PHY-aided MAC. IEEE Transactions on Wireless Communications, 14(2), 670-684.

Ksentini, A., \& Nikaein, N. (2017). Toward enforcing network slicing on RAN: Flexibility and resources abstraction. IEEE Communications Magazine, 55(6), 102-108.

Ksentini, A., Frangoudis, P. A., Amogh, P. C., \& Nikaein, N. (2018). Providing low latency guarantees for slicing-ready 5G systems via two-level MAC scheduling. IEEE Network, 32(6), 116-123.

Labiod, M. A., Gharbi, M., Coudoux, F. X., Corlay, P., \& Doghmane, N. (2019a). Cross-layer scheme for low latency multiple description video streaming over Vehicular Ad-hoc NETworks (VANETs). AEU-International Journal of Electronics and Communications, 104, 23-34.

Labiod, M. A., Gharbi, M., Coudoux, F. X., Corlay, P., \& Doghmane, N. (2019b). Enhanced adaptive crosslayer scheme for low latency HEVC streaming over Vehicular Ad-hoc Networks (VANETs). Vehicular communications, 15, 28-39.

Lähetkangas, E., Pajukoski, K., Vihriälä, J., Berardinelli, G., Lauridsen, M., Tiirola, E., \& Mogensen, P. (2014, June). Achieving low latency and energy consumption by 5G TDD mode optimization. In 2014 IEEE International Conference on Communications Workshops (ICC) (pp. 1-6). IEEE. 
Levanen, T., Pirskanen, J., \& Valkama, M. (2017). Low-latency Radio-interface Perspectives for Small-cell 5G Networks. Towards 5G: Applications, Requirements and Candidate Technologies, 275.

Levanen, T., Pirskanen, J., Koskela, T., Talvitie, J., \& Valkama, M. (2014, June). Low latency radio interface for $5 \mathrm{G}$ flexible TDD local area communications. In 2014 IEEE International Conference on Communications Workshops (ICC) (pp. 7-13). IEEE.

Li, Q., Wu, G., Papathanassiou, A., \& Mukherjee, U. (2016). An end-to-end network slicing framework for $5 \mathrm{G}$ wireless communication systems. arXiv preprint arXiv:1608.00572.

Liang, Y., Li, B., Yang, M., Zuo, X., Yan, Z., \& Xue, Q. (2016, August). MAC protocol framework for 5G mmWave backhaul network. In 2016 IEEE International Conference on Signal Processing, Communications and Computing (ICSPCC) (pp. 1-6). IEEE.

Ma, T., Hu, F., \& Ma, M. (2017, November). Fast and efficient physical layer authentication for $5 \mathrm{G} \mathrm{HetNet}$ handover. In 2017 27th International Telecommunication Networks and Applications Conference (ITNAC) (pp. 1-3). IEEE.

Mathur, S., Saha, D., \& Raychaudhuri, D. (2016, October). Cross-layer MAC/PHY protocol to support IoT traffic in 5G: poster. In Proceedings of the 22nd Annual International Conference on Mobile Computing and Networking (pp. 467-468).

Moreira, C. M., Kaddoum, G., \& Bou-Harb, E. (2018, May). Cross-layer authentication protocol design for ultra-dense 5G HetNets. In 2018 IEEE International Conference on Communications (ICC) (pp. 1-7). IEEE.

Papaioannou, S., Kalfas, G., Vagionas, C., Mitsolidou, C., Maniotis, P., Miliou, A., \& Pleros, N. (2018, January). $5 \mathrm{G}$ small-cell networks leveraging optical technologies with mm-wave massive MIMO and MT-MAC protocols. In Broadband Access Communication Technologies XII (Vol. 10559, p. 105590B). International Society for Optics and Photonics.

Pocovi, G., Soret, B., Pedersen, K. I., \& Mogensen, P. (2017, May). MAC layer enhancements for ultrareliable low-latency communications in cellular networks. In 2017 IEEE International Conference on Communications Workshops (ICC Workshops) (pp. 1005-1010). IEEE.

Raviraj, P., Sharif, H., Hempel, M., \& Ci, S. (2005, August). MOBMAC-an energy efficient and low latency MAC for mobile wireless sensor networks. In 2005 Systems Communications (ICW'05, ICHSN'05, ICMCS'05, SENET'05) (pp. 370-375). IEEE.
Rony, R. I., Lopez-Aguilera, E., \& Garcia-Villegas, E. (2018, December). Optimization of $5 \mathrm{G}$ fronthaul based on functional splitting at PHY layer. In 2018 IEEE Global Communications Conference (GLOBECOM) (pp. 1-7). IEEE.

Rost, P., Mannweiler, C., Michalopoulos, D. S., Sartori, C., Sciancalepore, V., Sastry, N., ... \& Aziz, D. (2017). Network slicing to enable scalability and flexibility in $5 \mathrm{G}$ mobile networks. IEEE Communications magazine, 55(5), 72-79.

Roth-Mandutz, E., Waswa, A. M., \& Mitschele-Thiel, A. (2017, November). Capacity optimization for ultrareliable low-latency communication in $5 \mathrm{G}$-The SON perspective. In 2017 13th International Conference on Network and Service Management (CNSM) (pp. 1-8). IEEE.

Sachs, J., Wikstrom, G., Dudda, T., Baldemair, R., \& Kittichokechai, K. (2018a). 5G radio network design for ultra-reliable low-latency communication. IEEE network, 32(2), 24-31.

Sachs, J., Andersson, L. A., Araújo, J., Curescu, C., Lundsjö, J., Rune, G., ... \& Wikström, G. (2018b). Adaptive 5G low-latency communication for tactile internet services. Proceedings of the IEEE, 107(2), 325-349.

Santoyo-González, A., \& Cervelló-Pastor, C. (2018). Latency-aware cost optimization of the service infrastructure placement in $5 \mathrm{G}$ networks. Journal of Network and Computer Applications, 114, 29-37.

Sayadi, M., Rodríguez, S., Olmos, J. J. V., \& Monroy, I. T. (2018). Latency and bit-error-rate evaluation for radioover-ethernet in optical fiber front-haul networks. Optical Switching and Networking, 27, 88-92.

Shreevastav, R., \& Carbajo, R. S. (2016, October). Dynamic RLC mode based upon link adaptation to reduce latency and improve throughput in cellular networks. In 2016 IEEE 7th Annual Ubiquitous Computing, Electronics \& Mobile Communication Conference (UEMCON) (pp. 1-6). IEEE.

Siddiqui, M. S., Legarrea, A., Escalona, E., Parker, M. C., Koczian, G., Walker, S. D., ... \& Ulbricht, M. (2016). Hierarchical, virtualised and distributed intelligence $5 \mathrm{G}$ architecture for low-latency and secure applications. Transactions on Emerging Telecommunications Technologies, 27(9), 1233-1241.

Wang, Y., Xu, C., He, Y., \& Zhou, Z. (2019, June). Low-Complexity Cross-Layer Resource Allocation for Low-Latency D2D-Based Relay Networks. In 2019 15th International Wireless Communications \& Mobile Computing Conference (IWCMC) (pp. 1619-1624). IEEE.

Yang, N., Wang, L., Geraci, G., Elkashlan, M., Yuan, J., \& Di Renzo, M. (2015). Safeguarding 5G wireless communication networks using physical layer security. IEEE Communications Magazine, 53(4), 20-27. 
Zafar, W., \& Khan, B. M. (2017). A reliable, delay bounded and less complex communication protocol for multicluster FANETs. Digital Communications and Networks, 3(1), 30-38.

Zhang, S., Xu, X., Wu, Y., \& Lu, L. (2014, November). 5G: Towards energy-efficient, low-latency and highreliable communications networks. In 2014 IEEE international conference on communication systems (pp. 197-201). IEEE.
Zhang, S., Gu, R., Ji, Y., Bai, L., Ma, Z., \& Chen, A. (2019). Efficient polling cycle adaptive passive optical network for low-latency $5 \mathrm{G}$ fronthaul. Optical Switching and Networking, 33, 122-130.

Zheng, G., Gao, Y., Fu, J., \& Tang, S. (2010, April). An energy efficient and low latency MAC protocol for multi-hop wireless sensor networks. In 2010 International Conference on Communications and Mobile Computing (Vol. 3, pp. 224-228). IEEE. 